\title{
Pericardial and serous effusions in primary hypothyroidism
}

\begin{abstract}
Pericardial effusion is frequently the sole serous effusion but pleural effusion and ascites are occasionally encountered in primary hypothyroidism, but the combination of all three in the same patient is extremely rare. Pericardial effusion is usually associated with myocardial dysfunction, non-specific E.C.G. changes, ST-T waves changes, sinus bradycardia but cardiac tamponade seldom occurs. ${ }^{1,2}$

In our study of 22cases of Primary hypothyroid women who have been diagnosed clinically and hormonally assayed. We could demonstrate a considerable relationship between cases with serous effusion (the wet hypothyroidism) in contrast to dry cases where serous effusion are not demonstrable, and fluid retaining activity with other subtle endocrine abnormalities which could be detected in those patients and their families.
\end{abstract}

Volume 6 Issue I - 2018

Saadi JS AlJadir

College of Medicine, Babylon University Formerly, Iraq

Correspondence: Saadi JS AlJadir, Babylon University, PO Box 498 Nassiriya,Thi Qar, Iraq, Email saljadir5 I@gmail.com

Received: December 02, 2017| Published: February 05, 2018

\section{Setting}

Marjan Teaching Hospital, College of Medicine, Babylon University, Babylon.

\section{Study objective}

To define primary hypothyroid patients into two distinctive categories according to the presence or absence of clinical demonstrable serous effusion and determination of the etiology and the contributory risk factor for the presence or the absence of serous fluid and edema fluid accumulation.

\section{Patients and methods}

22 women were studied. They have been referred from consultation clinic of Marjan Teaching Hospital and private clinic. Their age is ranging from 26-62years. Their mean age is 42years. The period between Oct 1994-Nov 1995.

\section{The following steps were undertaken}

i. Clinical evaluation

ii. Laboratory screening and investigations

Plain chest x-ray, E.C.G., Complete blood picture and E.S.R., Fasting blood sugar, Blood urea nitrogen, Serum electrolytes, serum protein, serum bilirubin, liver enzymes, serum alkaline phosphatase, cholesterol \& S. lipids and uric acid in some patients:

i. M-Mode and 2D Echo cardiogram and ultra sound study of cases with ascites.

ii. Hormonal study: radio- immunoassay, estimation of thyroid function (T3, T4, TSH, FIT), all of those patients have fulfilled the criteria of the diagnosis of primary thyroid failure which includes clinical evaluation and hormonal study.

\section{Clinical diagnosis}

Symptoms and signs of Primary hypothyroidism

\section{Hormonal study}

That confirms low T3 and T4 and high TSH or equivocal T3 and T4 but low FTI and high TSH (more or equal $10 \mathrm{mU} / \mathrm{ml}$ ). ${ }^{3,6}$ Those patients had received Eltroxin (L-thyroxin) in a dose of 100-200microgram per day in the morning on empty stomach after the diagnosis is well established.

Follow up of cases was carried regularly and most of those patients were visiting the clinic regularly.

\section{Clinical presentation}

General: in the early phase of the disease, the symptoms are nonspecific and may be passed unnoticed by the patients and the physicians. ${ }^{2}$ symptoms of primary thyroid failure could be attributed to deceleration of basal metabolic rate and to accumulation of deposition of connective tissues components (like glycosaminoglycans, hyaluronic acid etc....) and other mucopolysaccharides in the vocal cord, oropharynx and subcutaneous tissues. ${ }^{1,3}$

\section{General appearance}

Most of the patients exhibit dull expressionless faces, sparse and loss of hair, periorbital puffiness, and the voice is hoarse and deep, large tongue, pale dry, coarse and cold skin particularly in older subject, thyroid tissues is not palpable in most cases of primary thyroid failure except in goitrous primary hypothyroidism. ${ }^{1,2}$

\section{Cardiovascular system}

Most patients manifest bradycardia, systolic blood pressure is generally reduced, while diastolic blood pressure is usually elevated, that might be attributed to increase in the peripheral vascular resistance, heart size is increased and both myocardial dilatation and pericardial effusion are contributory factors, cardiac output is almost exclusively reduced.

\section{Nervous system}

There is deterioration in hearing, memory and intellectual abilities. A variety of psychiatric manifestations could be exhibited by many 
patients and called Myxedema Madness. Some patients present by cerebellar ataxia and most of the patients suffer from obstructive sleep apnea. There is abnormal response to tendon jerk in form of delay relaxation (hang up reflex), most of the patients suffer from muscle cramp and Carpal Tunnel Syndrome. ${ }^{1-3}$

\section{Gastrointestinal tract}

Appetite is usually declined although weight gain is marked and this is particularly attributed to low basal metabolism and fluid retention, most of the patients are constipated and there is a dynamic ileus that may occur producing megacolon or intestinal obstruction. ${ }^{1-3}$

\section{Endocrine abnormalities}

There is increase in the prolactin activity and ADH and those lead to galactorrhea and dilutional hyponatremia respectively. There is abnormal metabolism of sex hormones binding protein and this leads to menstrual abnormalities usually in form of menorrhagia, if the patient is left untreated for long time, and submitted to extreme cold, infection, surgery and anesthesia patient may pass into coma..$^{1,3,4}$ Myxedema come is a serious condition that mandates urgent medical intervention, otherwise mortality might score from $30-40 \%$.

\section{Skin changes}

The skin is thickened and shows a tendency to scale, the cutaneous thickening is due to mucinous infiltration which has a characteristics histological staining due to the increased contents of mucopolysaccharids, these changes if they are fully developed, state is called myxedema and gives its name to the syndrome of severe thyroid deficiency in adults. ${ }^{1-3}$

\section{Discussion}

Pericardial effusion is a frequent accompaniment of primary hypothyroid disease, pleural effusion is occasionally present and ascites with moderate quantity is less frequently detected in those patients and there may be special site of edema. ${ }^{3,4,8,9}$ Women are affected more than men. The mechanisms that underlying serous collection are not well established, but there are hypothetical links between primary thyroid failure and fluid accumulation in different serous cavities and body compartments. ${ }^{4,8,9}$

a. Renin-Angiotensin-Aldosterone system. ${ }^{4,5}$

b. Renal Dopamine secretion: decrease renal dopamine synthesis leads to sodium and water retention. ${ }^{5}$.

c. Increase Capillary permeability.,

d. Prolactin and ADH secretion and alteration in the metabolism of sex hormones and failure of handling water load like SIADH. ${ }^{1-3}$

All these factors may interplay together to produce the florid clinical picture to what could be called (Wet hypothyroidism), fluid is invariably transudate (protein content of less than $1.5 \mathrm{~g} / \mathrm{L}$ ). Presence of pericardial effusion does not correlate to myocardial dysfunction which accompanies other features; sinus bradycardia, ST-T waves changes, which resolve efficiently after thyroxine therapy, effusions and edema will clear up soon after optimal therapeutic doses of thyroxine, and maintenance of euthyroid status..$^{3-5}$

Pleural effusion may be present on one side of pleural space but both sides could be involved, the effusion is usually modest in quantity and is not usually associated with respiratory embarrassment. Ascites is less commonly encountered than other effusions. Most patients, which Myxedema develop edema in special sites (Periorbital and Pretibial). ${ }^{1,2}$

Fluid retention in primary hypothyroid disease is quite differing than those edema in conditions where there is augmented hydrostatic pressure like congestive cardiac failure or decrease oncotic pressure as in nephritic syndrome as a critical concept in the management, while thyroxine therapy can clear up fluid retention in primary thyroid failure without additional measures. 5,7

\section{Results}

From the 22women with primary hypothyroidism which were studied. 9 of them are having demonstrable serous effusions, particularly pericardial, pleural and occasionally ascites, and those serous effusions were confirmed by plain x-ray, echocardiography study and the ultrasound of the abdomen, and those patients were being treated effectively by thyroxine replacement.

The result was dramatic and further study had revealed no serous effusions in the sites that were previously detected. E.C.G changes recover to normal, thyroid hormonal study has been done and nearly euthyroid states were achieved for all the patients.

We reviewed retrospective history, family history of the two groups and we could recover interesting findings between wet cases of primary hypothyroidism and fluid retaining activity in those women and their families as well as the presence of other subtle endocrine abnormalities mainly obesity ( $25 \%$ weight gain above the standard body weight). Diabetes Mellitus and coexisting thyroid disease whether hypothyroidism or hyperthyroidism.

Patients with fluid retention and idiopathic edema have been screened for the clinical criteria essential for the diagnosis of fluid retention syndrome in women:

i. Non-dependent edema and fluid retention

ii. Autonomic over activity that involves bowel and bladder.

iii. Affective disorder in form of fatigue mood changes, anxiety and depression. ${ }^{5,7}$

\section{Acknowledgements}

None.

\section{Conflicts of interest}

None.

\section{Funding}

None.

\section{References}

1. Harrison's: Principles of Internal Medicine. $12^{\text {th }}$ edn. McGraw Hill Book Company, USA. 1995.

2. Cecil: Text Book of Medicine. Wyngarden \& Smith. W.B. Saunders International, $18^{\text {th }}$ edn.

3. Williams RB. Text Book of Endocrinology. London. W.B. Sanders, UK. 1974 
4. Al-Kader AA, Aber GM. The Relationship between Idiopathic edema Syndrome and Subclinical hypothyroidism. Clin Endocrinol (Oxf). 1979;10(3):271-279.

5. Dunnigan MG. Management of the fluid retention syndrome in women Hospital Update. 1990;653-663.

6. William FS, Lockwood DH. Endocrine diagnosis, clinical and laboratory approach. Little, Brown \& Company. Boston, USA. 1983.
7. Dunnigan MG. Recognition and management of the fluid retention. (Idiopathic or cyclical edema) and premenstrual syndromes in: $\mathrm{Mc}$ Naughton Medical gynecology. Edinburgh: Blackwell scientific, UK. $1985 ; 27-55$.

8. Streeten DHP. Idiopathic edema: pathogenesis, clinical features, and treatment. Endocrinol Metab Clin North Am. 1987;24(3):531-547.

9. Edwards OM, Bayliss RIS. Idiopathic edema of women QJ. Med ns XLV. $2000 ; 125-144$ 